\title{
Note on the Regularity of Nonadditive Measures
}

\author{
Toshikazu Watanabe, Syuji Yamada, and Tamaki Tanaka \\ Graduate School of Science and Technology, Niigata University, 8050 Ikarashi 2-no-cho, Nishi-ku, Niigata 950-2181, Japan
}

Correspondence should be addressed to Toshikazu Watanabe; wa-toshi@mti.biglobe.ne.jp

Received 6 June 2013; Revised 25 July 2013; Accepted 25 July 2013

Academic Editor: Wei-Shih Du

Copyright (C) 2013 Toshikazu Watanabe et al. This is an open access article distributed under the Creative Commons Attribution License, which permits unrestricted use, distribution, and reproduction in any medium, provided the original work is properly cited.

We consider the regularity for nonadditive measures. We prove that the non-additive measures which satisfy Egoroff's theorem and have pseudometric generating property possess Radon property (strong regularity) on a complete or a locally compact, separable metric space.

\section{Introduction}

The relations of continuity and regularity of nonadditive measures are considered in several papers [1-4]. In [5], Li et al. investigated the regularity in nonadditive measures. They proved that the null-additive fuzzy measures possess a Radon property (strong regularity) on a complete metric space. In [6], Kawabe also investigated the regularity in fuzzy measures taking value in Riesz spaces. He proved that every weakly null-additive Riesz space valued fuzzy measure on a complete or a locally compact, separable metric space is Radon, provided that the Riesz space has the multiple Egoroff property.

On the other hand Li and Mesiar [7] proved the regularity of nonadditive monotone measures. They proved that the equivalence condition of Egoroff's theorem implies regularity for the nonadditive measures by using pseudometric generating property of a set function. For information on real valued nonadditive measures, see [8-10].

In this paper, as notes, we prove that Egoroff's theorem implies Radon property (strong regularity) for nonadditive measures which have pseudometric generating property on a complete or a locally compact, separable metric space.

\section{Preliminaries}

Let $R$ be the set of real numbers and $N$ the set of natural numbers. In what follows, let $(X, \mathscr{F})$ be a measurable space.
Definition 1. A set function $\mu: \mathscr{F} \rightarrow R$ is called a nonadditive measure if it satisfies the following two conditions:

(1) $\mu(\emptyset)=0$,

(2) if $A, B \in \mathscr{F}$ and $A \subset B$, then $\mu(A) \leq \mu(B)$.

Definition 2. Let $\mu: \mathscr{F} \rightarrow R$ be a nonadditive measure.

(1) $\mu$ is said to be continuous from above if for any $\left\{A_{n}\right\} \subset$ $\mathscr{F}$ and $A \in \mathscr{F}$ satisfying $A_{n} \searrow A$ and there exists $n_{0}$ with $\mu\left(A_{n_{0}}\right)<\infty$ it holds that $\lim _{n \rightarrow \infty} \mu\left(A_{n}\right)=\mu(A)$.

(2) $\mu$ is said to be continuous from below if for any $\left\{A_{n}\right\} \subset$ $\mathscr{F}$ and $A \in \mathscr{F}$ satisfying $A_{n} \nearrow A$ it holds that $\lim _{n \rightarrow \infty} \mu\left(A_{n}\right)=\mu(A)$.

(3) $\mu$ is said to be fuzzy measure if it is continuous from above and below.

(4) $\mu$ is said to be strongly order continuous if it is continuous from above at measurable sets of measure 0 ; that is, for any $\left\{A_{n}\right\} \subset \mathscr{F}$ and $A \in \mathscr{F}$ satisfying $A_{n} \searrow A$ and $\mu(A)=0$ it holds that $\lim _{n \rightarrow \infty} \mu\left(A_{n}\right)=$ 0 .

(5) $\mu$ is said to be weakly null-additive if $\mu(A \cup B)=0$ whenever $A, B \in \mathscr{F}$ and $\mu(A)=\mu(B)=0$.

(6) $\mu$ has property $(S)$ if for any sequence $\left\{A_{n}\right\} \subset \mathscr{F}$ with $\lim _{n \rightarrow \infty} \mu\left(A_{n}\right)=0$ there exists a subsequence $\left\{A_{n_{k}}\right\}$ such that $\mu\left(\cap_{i=1}^{\infty} \cup_{k=i}^{\infty} A_{n_{k}}\right)=0$; see [11]. 
(7) $\mu$ is said to be autocontinuous from above if $\lim _{n \rightarrow \infty} \mu\left(A \cup B_{n}\right)=\mu(A)$ for each $A \in \mathscr{F}$ and $\left\{B_{n}\right\} \subset \mathscr{F}$ with $\lim _{n \rightarrow \infty} \mu\left(B_{n}\right)=0$.

(8) $\mu$ is said to be autocontinuous from below if $\lim _{n \rightarrow \infty} \mu\left(A \backslash B_{n}\right)=\mu(A)$ for each $A \in \mathscr{F}$ and $\left\{B_{n}\right\} \subset \mathscr{F}$ with $\lim _{n \rightarrow \infty} \mu\left(B_{n}\right)=0$.

(9) $\mu$ is said to be autocontinuous if it is autocontinuous from above and below.

Definition 3. Let $\mu: \mathscr{F} \rightarrow R$ be a nonadditive measure.

(1) A double sequence $\left\{A_{m, n}\right\} \subset \mathscr{F}$ is said to be a $\mu$ regulator if it satisfies the following two conditions:

(D1) $A_{m, n} \supset A_{m, n^{\prime}}$ whenever $n \leq n^{\prime}$,

(D2) $\mu\left(\cup_{m=1}^{\infty} \cap_{n=1}^{\infty} A_{m, n}\right)=0$.

(2) $\mu$ satisfies the Egoroff condition if for any $\mu$-regulator $\left\{A_{m, n}\right\}$ and for every $\varepsilon>0$ there exists a sequence $\left\{n_{m}\right\}$ of natural numbers such that $\mu\left(\cup_{m=1}^{\infty} A_{m, n_{m}}\right)<\varepsilon$.

Remark 4. A nonadditive measure $\mu$ satisfies the Egoroff condition if (and only if) for any double sequence $\left\{A_{m, n}\right\} \subset \mathscr{F}$ satisfying (D2) and the following (D1') it holds that for every $\varepsilon>0$ there exists a sequence $\left\{n_{m}\right\}$ of natural numbers such that $\mu\left(\cup_{m=1}^{\infty} A_{m, n_{m}}\right)<\varepsilon$ :

$\left(\mathrm{D}^{\prime}\right) A_{m, n} \supset A_{m^{\prime}, n^{\prime}}$ whenever $m \geq m^{\prime}$ and $n \leq n^{\prime}$.

\section{Compact Measure and Regularity of Measure}

In this section, we pick up several results for compact nonadditive measures and regularity of measures.

Definition 5. Let $\mu: \mathscr{F} \rightarrow R$ be a nonadditive measure.

(1) A nonempty family $\mathscr{K}$ of subsets of $X$ is called a compact system if for any sequence $\left\{K_{n}\right\} \subset \mathscr{K}$ with $\cap_{n=1}^{\infty} K_{n}=\emptyset$ there is $n_{0} \in N$ such that $\cap_{n=1}^{n_{0}} K_{n}=\emptyset$; see [12].

(2) We say that $\mu$ is compact if there exists a compact system $\mathscr{K}$ such that for each $A \in \mathscr{F}$ there are sequences $\left\{K_{n}\right\} \subset \mathscr{K}$ and $\left\{B_{n}\right\} \subset \mathscr{F}$ such that $B_{n} \subset$ $K_{n} \subset A$ for all $n \in N$ and $\lim _{n \rightarrow \infty} \mu\left(A \backslash B_{n}\right)=0$.

Remark 6. (1) The family of all compact subsets of a Hausdorff space is a compact system.

(2) The family of all finite unions of sets in a compact system is also compact [13, Lemma 1.4]. Therefore, in (2) of the above definition, the compact system $\mathscr{K}$ and the sequences $\left\{K_{n}\right\} \subset \mathscr{K}$ and $\left\{B_{n}\right\} \subset \mathscr{F}$ may be chosen so that $\mathscr{K}$ is closed for finite unions and both $\left\{K_{n}\right\}$ and $\left\{B_{n}\right\}$ are increasing.

By [6, Theorem 1], the following result follows.

Theorem 7. Let $\mu: \mathscr{F} \rightarrow R$ be a nonadditive measure. If $\mu$ is compact and autocontinuous, then it is continuous from above and below.
Proof. Since $\mu$ is compact and autocontinuous, by [6, Theorem 1], the assertion follows.

In what follows, let $(X, d)$ be a metric space. Denote by $\mathscr{B}(X)$ the $\sigma$-field of all Borel subsets of $X$, that is, the $\sigma$-field generated by the open subsets of $X$. A nonadditive measure defined on $\mathscr{B}(X)$ is called a nonadditive Borel measure on $X$.

Definition 8. $\mu$ is said to have pseudometric generating property if for each $\varepsilon>0$ there exists $\delta>0$ such that for any $A, B \in \mathscr{B}(X), \mu(A) \vee \mu(B)<\delta$ implies $\mu(A \cup B)<\varepsilon$.

Proposition 9. If $\mu$ satisfies pseudometric generating property, then it is weakly null-additive.

Proof. It is easy to see from the definition.

Definition 10. Let $\mu: \mathscr{B}(X) \rightarrow R$ be a nonadditive Borel measure on $X$.

$\mu$ is called regular if for any $\varepsilon>0$ and $A \in \mathscr{B}(X)$, there exist a closed set $F_{\varepsilon}$ and an open set $G_{\varepsilon}$ such that $F_{\varepsilon} \subset A \subset G_{\varepsilon}$ and $\mu\left(G_{\varepsilon} \backslash F_{\varepsilon}\right)<\varepsilon$.

Li and Mesiar [7] also investigated the regularity on monotone measures. The following follows.

Lemma 11. Let $X$ be a metric space and $\mu: \mathscr{B}(X) \rightarrow R a$ nonadditive Borel measure on $X$. If $\mu$ has the Egoroff condition and pseudometric generating property, then $\mu$ is regular.

Corollary 12. Let $X$ be a metric space and $\mu: \mathscr{B}(X) \rightarrow R a$ nonadditive Borel measure on $X$. If $\mu$ has property $(S)$, is strong order continuous, and is weakly null-additive, then $\mu$ is regular.

By Li and Yasuda [14, Theorem 1], we also have the following.

Corollary 13. Let $X$ be a metric space. If $\mu: \mathscr{B}(X) \rightarrow R$ is weakly null-additive fuzzy Borel measure on $X$, then it is regular. Moreover if $\mu$ is null-additive, we have

$$
\begin{aligned}
\mu(A) & =\sup \{\mu(F) \mid F \subset A, F \text { is closed set }\} \\
& =\inf \{\mu(G) \mid G \supset A, G \text { is open set }\} .
\end{aligned}
$$

Corollary 13 above is a special case of [6, Theorem 5] and [15, Theorem 3].

For more information on regularity of nonadditive measures, see $[5,6]$.

\section{Radon Measure}

In this section, as main results, if we assume that a nonadditive Borel measure satisfies the equivalence condition of Egoroff's theorem and pseudometric generating property on a complete or a locally compact, separable metric space, then it is Radon.

Definition 14. Let $\mu$ be a nonadditive Borel measure on $X$.

(1) $\mu$ is said to be Radon (strongly regular) if for each $A \in \mathscr{B}(X)$ there are sequences $\left\{K_{n}\right\}_{n \in N}$ of compact 
sets and $\left\{G_{n}\right\}_{n \in N}$ of open sets such that $K_{n} \subset A \subset G_{n}$ for all $n \in N$ and $\lim _{n \rightarrow \infty} \mu\left(G_{n} \backslash K_{n}\right)=0$.

(2) $\mu$ is said to be tight if there is a sequence $\left\{K_{n}\right\}_{n \in N}$ of compact sets such that $\lim _{n \rightarrow \infty} \mu\left(X \backslash K_{n}\right)=0$.

Remark 15. Sequences of sets in the above definition may be chosen so that $\left\{G_{n}\right\}_{n \in N}$ is decreasing, while $\left\{F_{n}\right\}_{n \in N}$ and $\left\{K_{n}\right\}_{n \in N}$ are increasing.

Proposition 16. Let $X$ be a Hausdorff space. Let $\mu$ be a nonadditive Borel measure on $X$ which is weakly null-additive and strongly order continuous. Then, the following two conditions are equivalent:

(i) $\mu$ is Radon (strongly regular),

(ii) $\mu$ is regular and tight.

Proof. See [6, Proposition 2].

It is known that every finite Borel measure on a complete or a locally compact, separable metric space is Radon; see [16, Theorem 3.2] and [17, Theorems 6 and 9, Chapter II, Part I]. Its counterpart in nonadditive measure theory can be found in [5, 9, Theorem 1, Lemma 2], which states that every Borel fuzzy measure on a complete separable metric space is tight, so that it is Radon if it is null-additive; see also [3, Theorem 2.3]. The following two theorems contain those previous results; see also [18, Theorem 12].

Theorem 17. Let $X$ be a complete separable metric space and $\mu: \mathscr{B}(X) \rightarrow R$ a nonadditive Borel measure on $X$. If $\mu$ is weakly null-additive and satisfies the Egoroff condition, then it is tight. Moreover, if $\mu$ has pseudometric generating property and satisfies the Egoroff condition, then it is Radon.

To prove the theorem, we need the following; see [7, Proposition 3.7].

Proposition 18. Let $\mu: \mathscr{F} \rightarrow R$ be a nonadditive measure. Then (i) implies (ii).

(i) $\mu$ is weakly null-additive and satisfies the Egoroff condition.

(ii) For each $\varepsilon>0$ and double sequence $\left\{A_{m, n}\right\} \subset \mathscr{F}$ satisfying $A_{m, n} \searrow \emptyset$ as $n \rightarrow \infty$ for each $m \in N$, there exists a sequence $\left\{n_{m}\right\}$ of natural numbers such that $\mu\left(\cup_{m=1}^{\infty} A_{m, n_{m}}\right)<\varepsilon$.

Proof of Theorem 17. Since $\mu$ satisfies the Egoroff condition, by [19, Proposition 3], it is strongly order continuous. By Proposition 16 and Lemma 11, we have only to prove that $\mu$ is tight. Let $\left\{s_{i}\right\}_{i \in N}$ be a countable dense subset of $X$. For each $m, i \in N$, denote by $\overline{B_{m}\left(s_{i}\right)}$ the closed ball with center $s_{i}$ and radius $1 / m$. For each $m, n \in N$, put $A_{m, n}:=X \backslash \cup_{i=1}^{n} \overline{B_{m}\left(s_{i}\right)}$. Then, for any $\varepsilon>0$ and $m \in N$, we have $A_{m, n} \searrow \emptyset$, so that by Proposition 18, there exists a sequence $\left\{n_{m}\right\}$ of natural numbers such that

$$
\mu\left(\cup_{m=1}^{\infty} A_{m, n_{m}}\right)<\varepsilon .
$$

Put $P_{\varepsilon}:=\cap_{m=1}^{\infty} \cup_{i=1}^{n_{m}} \overline{B_{m}\left(s_{i}\right)}$. Then, each $P_{\varepsilon}$ is closed and totally bounded, so that it is compact. Since $X \backslash P_{\varepsilon}=\cup_{m=1}^{\infty} A_{m, n_{m}}$, it follows from (2) that $\mu\left(X \backslash P_{\varepsilon}\right)<\varepsilon$. Thus $\mu$ is tight.

Corollary 19. Let $X$ be a complete separable metric space and $\mu: \mathscr{B}(X) \rightarrow R$ a nonadditive Borel measure on $X$. If $\mu$ is weakly null-additive, strongly order continuous, and has property (S), then it is Radon.

Proof. It follows from Theorem 17 since $\mu$ has pseudometric generating property [7, Proposition 5.1] and satisfies the Egoroff condition [19, Proposition 2].

Corollary 20. Let $X$ be a complete separable metric space and $\mu: \mathscr{B}(X) \rightarrow R$ a fuzzy measure on $X$. If $\mu$ is weakly nulladditive, then it is Radon.

Proof. It follows from Theorem 17 since $\mu$ satisfies the Egoroff condition [7, Proposition 3.1] and it is regular [14, Theorem 1].

Remark 21. Corollary 20 above is a special case of [6, Theorem 5] and [15, Theorem 3].

Theorem 22. Let $X$ be a locally compact, separable metric space and $\mu: \mathscr{B}(X) \rightarrow R$ a nonadditive Borel measure on $X$. If $\mu$ is weakly null-additive and satisfies the Egoroff condition, then it is tight. Moreover, if $\mu$ has pseudometric generating property and satisfies Egoroff condition, then it is Radon.

Proof. By Lemma 11 and Proposition 16, we have only to prove the tightness of $\mu$. Denote by $\mathscr{H}$ the family of all open and relatively compact subsets of $X$. The local compactness of $X$ implies that $\mathscr{H}$ is an open cover of $X$. Since $X$ is strongly Lindelöf, that is, every open cover of any open subset of $X$ has a countable subcover [17, Proposition 3 and Theorem 6, Chapter II, Part I], there is a sequence $\left\{H_{m}\right\}_{m \in N} \subset \mathscr{H}$ such that $X=\cup_{m=1}^{\infty} H_{m}$. Put $K_{n}:=\cup_{m=1}^{n} \overline{H_{m}}$ for all $n \in N$, where $\bar{A}$ denotes the closure of a set $A$. Then $K_{n}$ is compact and $X \backslash K_{n} \searrow \emptyset$. Since $\mu$ is strongly order continuous [19, Proposition 3], $\lim _{n \rightarrow \infty} \mu\left(X \backslash K_{n}\right)=0$. Thus $\mu$ is tight.

Corollary 23. Let $X$ be a locally compact, separable metric space and $\mu: \mathscr{B}(X) \rightarrow R$ a nonadditive Borel measure on $X$. If $\mu$ is weakly-null-additive, strongly order continuous, and has property $(S)$, then $\mu$ is Radon.

Corollary 24. Let $X$ be a locally compact, separable metric space and $\mu: \mathscr{B}(X) \rightarrow R$ a fuzzy Borel measure on $X$. If $\mu$ is weaklly null-additive, then $\mu$ is Radon.

Remark 25. Corollary 24 above is a special case of [6, Theorem 6] and [15, Theorem 4].

\section{Acknowledgments}

The authors are grateful to the referees for their valuable suggestions and careful reading to revise this paper. The first author would like to express his hearty thanks to Professor 
Shizu Nakanishi. The first author also would like to express his hearty thanks to Professor Toshiharu Kawasaki and Professor Ichiro Suzuki for many valuable suggestions.

\section{References}

[1] Q. Jiang and H. Suzuki, "Fuzzy measures on metric spaces," Fuzzy Sets and Systems, vol. 83, no. 1, pp. 99-106, 1996.

[2] E. Pap, "Regular null additive monotone set functions," Univerzitet u Novom Sadu, vol. 25, no. 2, pp. 93-101, 1995.

[3] W. Congxin and H. Minghu, "On the regularity of the fuzzy measure on metric fuzzy measure spaces," Fuzzy Sets and Systems, vol. 66, no. 3, pp. 373-379, 1994.

[4] J. Wu and $\mathrm{C}$. Wu, "Fuzzy regular measures on topological spaces," Fuzzy Sets and Systems, vol. 119, no. 3, pp. 529-533, 2001.

[5] J. Li, M. Yasuda, and J. Song, "Regularity properties of nulladditive fuzzy measure on metric spaces," Lecture Notes in Artificial Intelligence, Springer, Berlin, Germany, vol. 3558, pp. 59-66, 2005.

[6] J. Kawabe, "The Alexandroff theorem for Riesz space-valued non-additive measures," Fuzzy Sets and Systems, vol. 158, no. 21, pp. 2413-2421, 2007.

[7] J. Li and R. Mesiar, "Lusin's theorem on monotone measure spaces," Fuzzy Sets and Systems, vol. 175, no. 1, pp. 75-86, 2011.

[8] D. Denneberg, Non-Additive Measure and Integral, Kluwer Academic, Dordrecht, The Netherlands, 2nd edition, 1997.

[9] E. Pap, Null-Additive Set Functions, Kluwer Academic, Dordrecht, The Netherlands, 1995.

[10] Z. Wang and G. J. Klir, Fuzzy Measure Theory, Plenum Press, New York, NY, USA, 1992.

[11] S. Qinghe, "Property (S) of fuzzy measure and Riesz's theorem," Fuzzy Sets and Systems, vol. 62, no. 1, pp. 117-119, 1994.

[12] E. Marczewski, "On compact measures," Fundamenta Mathematicae, vol. 40, pp. 113-124, 1953.

[13] J. Pfanzagl and W. Pierlo, Compact Systems of Sets, Lecture Notes in Mathematics, vol. 16, Springer, New York, NY, USA, 1966.

[14] J. Li and M. Yasuda, "Lusin's theorem on fuzzy measure spaces," Fuzzy Sets and Systems, vol. 146, no. 1, pp. 121-133, 2004.

[15] J. Kawabe, "Regularities of Riesz space-valued non-additive measures with applications to convergence theorems for Choquet integrals," Fuzzy Sets and Systems, vol. 161, no. 5, pp. 642650, 2010.

[16] K. R. Parthasarathy, Probability Measures on Metric Spaces, Academic Press, New York, NY, USA, 1967.

[17] L. Schwartz, Radon Measures on Arbitrary Topological Spaces and Cylindrical Measures, Oxford University Press, Oxford, UK, 1973.

[18] J. Kawabe, "Uniformity for weak order convergence of riesz space-valued measures," Bulletin of the Australian Mathematical Society, vol. 71, no. 2, pp. 265-274, 2005.

[19] T. Murofushi, K. Uchino, and S. Asahina, "Conditions for Egoroff's theorem in non-additive measure theory," Fuzzy Sets and Systems, vol. 146, no. 1, pp. 135-146, 2004. 


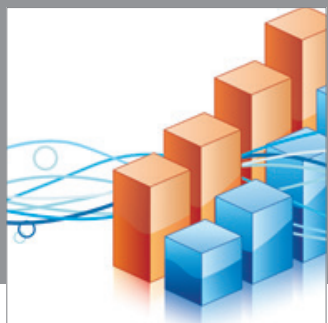

Advances in

Operations Research

mansans

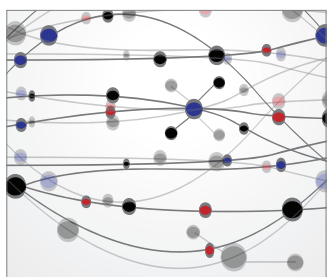

The Scientific World Journal
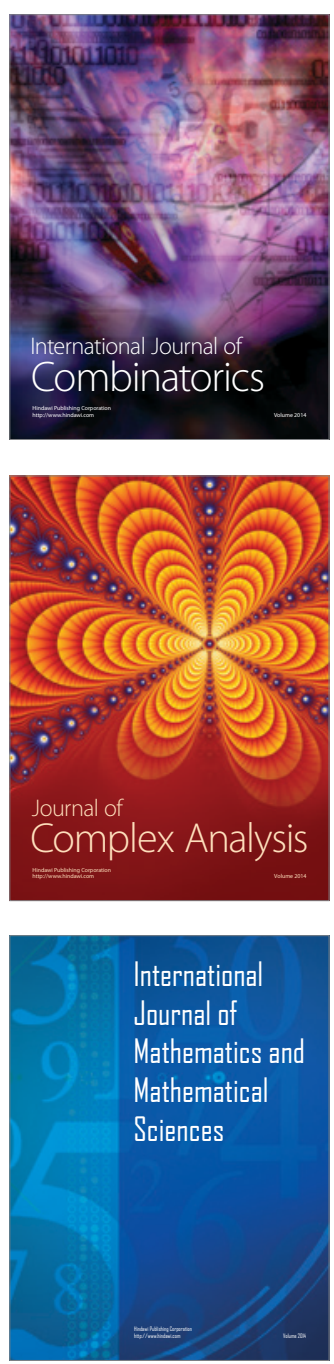
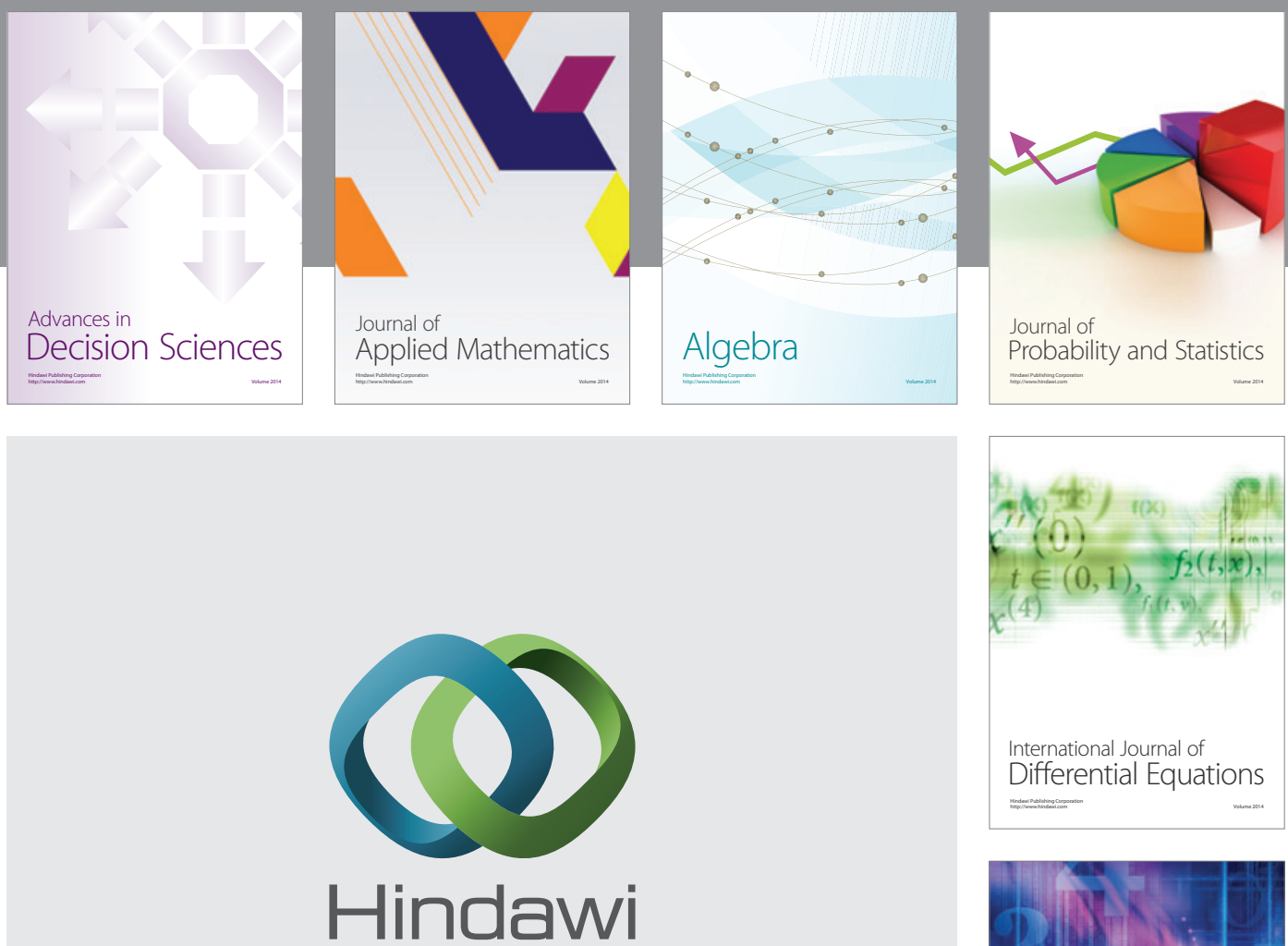

Submit your manuscripts at http://www.hindawi.com
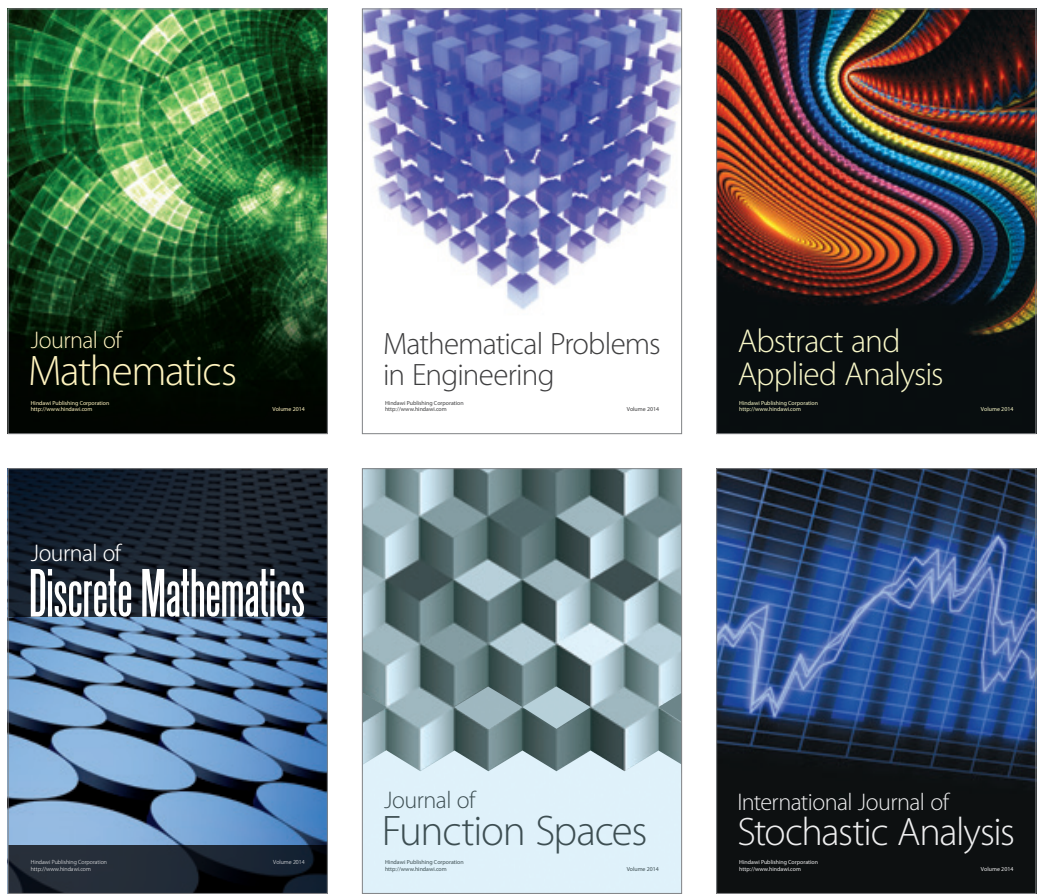

Journal of

Function Spaces

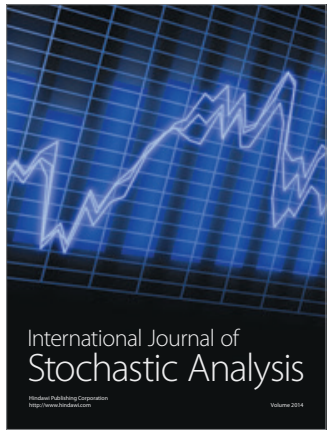

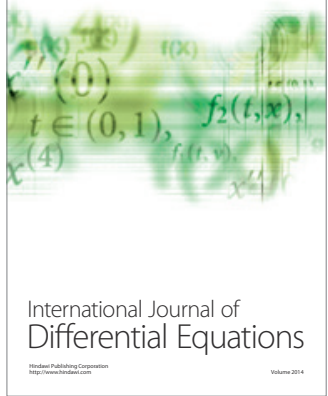
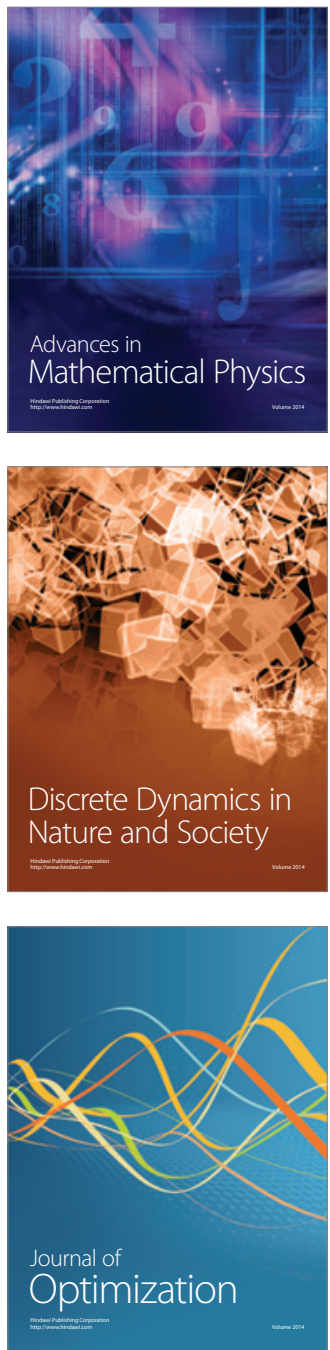